

\section{INDICE}

EDITORIAL PERSONAL ÁMBITOS

Apresentação do monográfico. Abordagem qualitativa: olhares e práticas transdisciplinares nas ciências antropossociais

Presentation of the monograph. Qualitative approach: transdisciplinary views and practices in anthroposocial sciences

Ronaldo Nunes Linhares, António Pedro Costa

MONOGRAFICOS MONOGRAPHS

Identidades femininas na rede: as crianças falam!

Female identities on line: children can speak

Marta Maria Azevedo Queiroz

$12-31$

Transição de cuidados de enfermagem: ISBAR na promoção da segurança dos

doentes - revisão scoping

Transition of nursing care: ISBAR in promoting patient safety - scoping review

Ana Rita Esteves Figueiredo, Teresa Maria Ferreira dos Santos Potra, Pedro Ricardo Martins Bernardes Lucas

$32-48$

Integración de elementos cualitativos y cuantitativos en metodología observacional

Integration of qualitative and quantitative elements in observational methodology

M. Teresa Anguera, Angel Blanco-Villaseñor, José Luis Losada, Pedro Sánchez-Algarra

49-70

Atos educativos com oficines de ecografias: uma investigação otobiográfica

Educational acts at echographie's cineliers: an otobiographic research

Silas Borges Monteiro, Anaise Avila Severo

Actuación de las políticas: política como texto y política como discurso

Action of policies: policy as text and policy as discourse

Mónica Rocío Barón

88-104 


\section{ÁMBITOS PERSONALES PERSONAL ÁMBITOS}

Un retrato de la cultura local a través del Periodismo cultural. Análisis comparado de Sevilla y Porto Alegre

A portrait of the local culture through cultural Journalism. Comparative analysis of Seville and Porto Alegre

\section{ARTÍCULOS ARTICLES}

Microsociología del profesor universitario

Microsociology of an university professor

Antonio Fernández Vicente

La pobreza y el discurso de los mass media. Un estudio de la prensa local argentina

Poverty and mass media 's discourse. A study of the Argentine local press

María del Rosario Sanchez, Silvia London

La comunicación no verbal en las elecciones andaluzas de 2018. Comparativa de Susana Díaz y Teresa Rodríguez en el debate de RTVE

Non-verbal communication in the Andalusian municipal elections of 2018. Comparison of Susana Díaz and Teresa Rodríguez in the electoral RTVE debate

María Hernández Herrarte, Patricia Zamora-Martínez

El infoentretenimiento en la televisión de pago, Movistar+ y el canal \#0.

El uso transmedia de sus contenidos de humor

Infotainment on pay television, Movistar+ and channel \# 0 . The transmedia use of its humorous content

Metodología y formación docente cuestiones claves para la integración de las TIC en la educación

Methodology and teacher training as a key issue for ICTs integration in Education 


\section{RESEÑAS REVIEWS}

Aquelarre. Mujeres en la cultura de masas

Coven. Women in mass culture

Regla Ismaray Cabreja Piedra

216-220

Transición ecosocial y principios éticos en el periodismo: una guía para la comunicación de nuevas narrativas

The Eco-social transition and ethical principles in journalism: a guide for the communication of new narratives

Amanda Salazar Torres

Narrativas ecofeministas y mapa de transición ecosocial para medios de comunicación Eco-feminist narratives and ecosocial transition map for the media 


\title{
Metodología y formación docente cuestiones claves para la integración de las TIC en la educación
}

\author{
Methodology and teacher training as a key issue for ICTs \\ integration in Education
}

\author{
Dra. Rebeca Suárez-Álvarez, Universidad Rey Juan Carlos, Camino del molino \\ s/n. 28943 Fuenlabrada, Madrid. España \\ rebeca.suarez@urjc.es | Orcid: https://orcid.org/0000-0002-0102-4472
}

Dra. Tamara Vázquez-Barrio, Universidad San Pablo CEU, Paseo Juan XXIII, 10. 28040 Madrid, España

tamarav@ceu.es | Orcid: http://orcid.org/0000-0003-2789-8554

\author{
Dra. Teresa Torrecillas Lacave, Universidad San Pablo CEU, Paseo Juan XXIII, \\ 10. 28040 Madrid, España \\ teresat@ceu.es | Orcid: http://orcid.org/0000-0001-6167-658X
}

DOI: https://dx.doi.org/10.12795/Ambitos.2020.i49.12

\begin{abstract}
Resumen
Las Administraciones Públicas son actores clave para la incorporación de las Tecnologías de la Información y la Comunicación (TIC) en los colegios. Son los organismos públicos educativos los que establecen los currículos escolares y los que deciden la dotación de recursos. Existe consenso sobre la necesidad de que sean las instituciones políticas las que apoyen, desde sus instancias, la incorporación de las TIC en la enseñanza. El propósito de la presente investigación se centra en conocer las acciones que plantean las políticas públicas para la integración TIC en la educación y
\end{abstract}


analizar si dichas iniciativas han logrado sus objetivos. La metodología utilizada es cualitativa mediante las técnicas de investigación de celebración de un panel de expertos y de 6 entrevistas en profundidad a directores y responsables TIC de centros educativos de Madrid. La conclusión es que los planes de las instituciones públicas para la inclusión digital se han centrado principalmente en cuestiones técnicas y han obviado las de carácter pedagógico que no depende de los dispositivos electrónicos utilizados, sino de los modelos didácticos aplicados. Expertos y equipos directivos consideran que la solución pasa por mejorar las infraestructuras tecnológicas de los colegios, pero sobre todo en invertir en metodologías que permitan a los profesores innovar en sus prácticas pedagógicas e incrementar la formación del profesorado en competencias y alfabetización digital para que puedan utilizar las TIC con finalidades educativas de manera solvente.

\section{Abstract}

Public administration is key agents for incorporation Information and Communication Technologies (ICT) in schools. School syllabuses are established by public entities and they decide resource endowment. There is consensus on the need to regarding public administration for support the incorporation of ITC in education. The aim of this research presents an overview of ICT integration policies in Spain education system and in order to establish whether they have achieved their objectives. The methodology used an expert panel of independent consultants and 6 in-depth interviews with directors and ICT managers of schools in Madrid. The research shows that integration policies have focused in technical issues and have ignored pedagogical philosophy. This educational practices do not depend on electronic devices but teaching models applied. Experts and management teams consider that the solution is to improve technological infrastructure of schools, but above all invest in methodologies that allow teachers to innovate in their pedagogical practices and increase teacher-training in competencies and digital literacy so they can use ICT with educational purposes in an effective way.

Palabras clave: administraciones públicas, proyectos TIC; Agenda Digital; competencias digitales; formación docente

Keywords: public administrations; ICT projects; Digital Agenda; digital competences; teacher education

\section{INTRODUCCIÓN}

El estudio de las políticas públicas llevadas a cabo por los gobiernos y su posterior análisis por parte de los científicos (Dror, 2017; Hill \& Hupe, 2014; Knill \& Tosun, 2012) ponen de manifiesto la responsabilidad del sector público acerca de "la calidad y las condiciones de ejercicio del sistema democrático contemporáneo, [...] cuyo objetivo fundamental es identificar los fundamentos de las políticas públicas y las condiciones, 
favorables o desfavorables, para su aplicación 'exitosa'" (Albuquerque, 2017, p. 1-2). La efectividad, calidad y utilidad unidas al presupuesto son los principales supuestos valorativos de las iniciativas impulsadas por las administraciones públicas. De hecho, el incremento del gasto público en educación se considera un indicador del compromiso de un gobierno con el desarrollo de habilidades y competencias (Bley, Hametner, Dimitrova, Ruech, De Rocchi, Gschwend \& Umpfenbach, 2018).

La implicación y apuesta de las entidades gubernamentales por la integración de las TIC en los centros escolares se revela necesaria (Kozma \& Isaacs, 2011); sin su apoyo, su incorporación se realizaría de forma episódica y respondería a la voluntad de algunos docentes y centros, pero no existiría un planteamiento general que englobe a todo el sistema educativo (Moreira, 2002). De hecho, en los últimos años ha llegado "a erigirse como una necesidad y como una herramienta de trabajo básica para el profesorado y el alumnado" (Fernández, 2010, p. 1). La UNESCO (2019) refrenda dicha relevancia y afirma que facilitan el acceso universal a la educación, reducen las diferencias en el aprendizaje, apoyan el desarrollo de los docentes, mejoran la calidad y la pertinencia del aprendizaje, refuerzan la integración y perfeccionan la gestión y administración de la educación. Además, le aportan valor añadido a las áreas tradicionales del currículo puesto que permiten desarrollar la comprensión y el uso crítico cognitivo y creativo (Gómez \& Macedo, 2010; McKnight, et al 2016; Sangra \& González-Sanmamed, 2011).

Los planes impulsados por los organismos públicos deberían garantizar la alfabetización digital de los menores. De este modo, podrían usar las TIC con sentido crítico, adquirirían competencias digitales centradas en habilidades comunicativas y en una mayor capacidad de análisis de la información a la que acceden (Fundación Telefónica, 2012); podrían, mediante las metodologías didácticas adecuadas, incrementar su aprendizaje, la búsqueda de información y el desarrollo de conocimientos (Tur-Porcar, 2017) así como potenciar simultáneamente el trabajo individual y las actividades colaborativas durante su formación (Courts, \& Tucker, 2012; Muñoz-Repiso, GómezPablos \& García, 2014).

La hipótesis se centra en dilucidar si el sistema educativo español ha conseguido integrar de manera efectiva las tecnológicas digitales en el proceso educativo gracias al impulso de los proyectos e iniciativas puestas en marcha por las administraciones públicas. Así, los objetivos de esta investigación se enfocan en conocer si dichos planes impulsados por los organismos públicos nacionales con el fin de integrar las TIC en los centros escolares han resultado eficaces. Para ello, se ha realizado un panel de expertos y seis entrevistas en profundidad a miembros de equipos directivos de centros educativos.

\section{REVISIÓN TEÓRICA Y PLANTEAMIENTO DEL PROBLEMA}

Los planes puestos en marcha en España emanan de las iniciativas planteadas por la Unión Europea. En diciembre de 1999, nació la Iniciativa Estratégica para el Desarrollo 
de la Sociedad de la Información "Info XXI" que se concretó con el nacimiento del "Plan de Acción 2001-2003 "Info XXI: La Sociedad de la información para todos”, presentado en enero de 2001. Este proyecto surge en respuesta a los objetivos planteados por el Plan de Acción eEurope - eEurope 2002 y eEurope 2005 - surgido de la Estrategia Lisboa, inaugurada en marzo del año 2000, y aprobado durante uno de los turnos de la Presidencia española del Consejo de la Unión Europea.

A continuación, en 2004, se inauguró el programa de actuaciones "España.es" con el objetivo de desarrollar la sociedad de la información dirigido a 17.500 centros, 420.000 profesores y 5.400 .000 millones de alumnos de centros públicos y concertados de las enseñanzas obligatorias (Primaria y ESO), Bachillerato y Formación Profesional. Cumplió parte de los objetivos "sobre todo lo que se refiere a la conexión de los centros educativos a Internet a través de la banda ancha y la dotación de redes de área local a casi el 100\% de los centros escolares" (Martín-Laborda, 2005, p. 13). Tras este proyecto, Red.es inaugura en septiembre de 2004 el proyecto "Centros Educativos Avanzados en el uso de las TIC" (redTIC) dirigido a integrar las TIC en el aula. García-Muñoz, Valiente, Barreiro, Díaz-Maroto y Rumayor $(2009$, p. 6) destacan de los resultados obtenidos por este plan que las valoraciones de los responsables TIC de los centros participantes "reflejaban que el uso habitual de las TIC en los centros educativos supuso cambios positivos en todos los agentes implicados".

En 2009, el Ministerio de Educación inicia el "Programa Escuela 2.0" que proponía conseguir que todos los estudiantes dispusiesen de un ordenador en sus aulas mediante la premisa "un ordenador por estudiante". El principal objetivo de este proyecto, que contó con una inversión de más de 98 millones de euros, era "la modernización de nuestro sistema educativo mediante la integración de las TIC" (Sanz, 2011). Pretendía transformar las aulas tradicionales en aulas digitales en las que los alumnos pudiesen estudiar con pizarras digitales y disponer de las infraestructuras tecnológicas y de la conectividad a Internet necesaria para desarrollar su día a día en la escuela. Su objetivo era digitalizar 14.000 aulas, formar a 20.000 profesores e informatizar a 400.000 alumnos de entre 10 y 13 años, dotándole de un ordenador portátil a cada uno con conexión a Internet para mejorar la calidad de la enseñanza, incorporar las nuevas tecnologías en la función docente y contrarrestar la brecha digital. Proyectaba dotar de un ordenador a cada alumno y formar, no solo en los aspectos tecnológicos, sino también en los aspectos metodológicos y sociales del uso de las TIC en el aula, a los profesores, tutores y a los responsables de tecnologías de cada centro (Ministerio de Ciencia y Tecnología, 2009). En este proyecto, que financió el Ministerio de Educación y las Comunidades Autónomas al 50\%, también estuvieron implicados los centros educativos, el profesorado, las empresas tecnológicas y de comunicación, las editoriales y empresas de software educativo y las propias familias de los alumnos. El Programa Escuela 2.0 tuvo una implantación desigual en las comunidades autónomas que lo pusieron en marcha (Area, Alonso, Correa, Del Moral, De Pablos, Paredes \& Valverde, 2014) y cada una lo ejecutó de forma diferente. 
En abril de 2011, coincidiendo con la crisis económica, la Secretaria de Estado de Educación, Formación Profesional y Universidades del Ministerio de Educación, Cultura y Deporte hizo públicos los recortes presupuestarios del sistema educativo español. Entre sus decisiones relacionadas con las medidas de política educativa anunció la supresión del Programa Escuela 2.0. y presentó, a grandes rasgos, un nuevo programa TIC, que aún hoy no se ha puesto en marcha. Para tomar esta decisión, el gobierno alegó argumentos aparentemente técnicos según distintos estudios que avalaban o evidenciaban la ineficacia o evaluación negativa del mismo (Moreira, 2012) pero el mismo autor se muestra crítico ante los argumentos utilizados por el gobierno para desactivar el plan y se pregunta "¿cuáles son esos estudios, dónde están, quién los firma? Confieso que no los conozco” (Moreira, 2012). El Programa Escuela 2.0 tiene buena intención en cuanto a acercar los ordenadores a los alumnos y ofrecer a los profesores materiales adaptados a las nuevas necesidades de sus clases, pero no les enseña cómo utilizarlas adecuadamente mediante una revisión profunda de las políticas y los métodos de enseñanza empleados.

Cuatro años más tarde, en febrero de 2013, derivada de la Agenda Digital Europea (European Commission, 2010), el Consejo de Ministros aprobó la Agenda Digital para España cuyo objetivo principal es desarrollar la economía y la sociedad digital en nuestro país (Ministerio de Industria, Comercio y Turismo, 2015). La Agenda Digital para España se articula mediante diez planes que se desarrollan en seis objetivos. De los diez planes, dos hacen referencia a las TIC y a la educación, pero en ambos casos desde un punto de vista técnico. El "Plan de servicios públicos digitales" plantea como actuación dotar a los centros educativos de banda ancha ultrarrápida en 2015. El "Plan de inclusión digital y empleabilidad" pretende conseguir que la mayoría de la población use Internet para alcanzar las metas europeas de inclusión digital y minimizar la brecha digital, pero no incluye ninguna acción concreta para la integración de las TIC en las escuelas. El sexto objetivo se centra en promover la inclusión, la alfabetización digital y la formación de nuevos profesionales TIC. Se divide en dos líneas de actuación. La primera pretende incorporar a la Sociedad de la Información a los colectivos más desfavorecidos y con menor nivel de utilización de Internet, y la segunda, busca actualizar el Catálogo Nacional de Cualificaciones Profesionales en materia de habilidades y capacitación TIC y maximizar la eficiencia en la gestión y asignación de los Fondos de Formación destinados a la formación continua en materia TIC.

En 2016, el gobierno actualizó en la Comisión de Industria del Congreso de los Diputados las principales líneas de actuación en materia de Agenda Digital (López \& Martínez, 2017) e identificó seis áreas de actuación prioritarias: despliegue de redes de nueva generación, derechos digitales de los ciudadanos, transformación digital de bienes y servicios, impulso al surgimiento de nuevas plataformas y servicios digitales, nuevos modelos de gestión de derechos y de negocio en los contenidos digitales y fiscalidad. Ninguna se centra en la integración de las TIC en el sistema escolar para mejorar los procesos de enseñanza-aprendizaje y desde la supresión en 2012 del Plan 
Escuela 2.0. no se ha promovido desde el Gobierno de España ningún proyecto similar y "prácticamente se han suprimido partidas económicas para la adquisición de recursos tecnológicos y de conectividad de los centros repercutiendo parte de los costes en las familias" (Area, et al., 2014, p. 31).

\subsection{Materiales y métodos}

Para lograr el objetivo planteado en la presente investigación se decidió aplicar metodología cualitativa a través de las dos técnicas de investigación: la celebración de un panel de expertos y la realización de seis entrevistas en profundidad a miembros de equipos directivos de centros educativos. Cada técnica plantea una serie de subobjetivos que soportaban los conocimientos necesarios para la construcción de la investigación.

El objetivo de la celebración de un panel de expertos fue analizar y valorar la revolución educativa (social, cognitiva y creativa) a la que estamos asistiendo con la incorporación de las TIC a las escuelas y a la vida de los estudiantes. El panel de expertos se circunscribe dentro de la técnica de investigación cualitativa de los focus group o grupos de discusión que permiten la obtención de información gracias a una mayor comprensión de las opiniones, actitudes y creencias de los usuarios (Ivankovich-Guillén \& Araya-Quesada, 2011; Krueger, 2014) con el objetivo de comprender los fenómenos sociales en términos de sus significados (Agius, 2013). Estas discusiones, diseñadas para obtener las percepciones sobre un área de interés particular, presentan dos sentidos. El primero centrado en el abordaje a fondo de un número de tópicos o dimensiones de estudio. Y el segundo, basado en la configuración misma del grupo que se realiza a partir de la identificación de algún tema prioritario compartido por las personas participantes (Murillo, 2010).

El panel congregó a los profesionales de distintas disciplinas que aúnan una amplia experiencia docente o que desarrollan su actividad en diversos ámbitos de la integración TIC de los centros escolares con una visión objetiva y pragmática de la implantación de las nuevas tecnológicas en las escuelas y colegios españoles. La selección de los participantes se realizó priorizando la interdisciplinariedad y la experiencia. Participaron un abogado especialista en Derecho de las Nuevas Tecnologías, un profesor y adjunto a la dirección de diferentes colegios durante su carrera profesional, el director de un gabinete psicopedagógico, un profesor con más de 22 años de experiencia y un director de Programas Formativos, Postgrado y Executive Education de la Escuela de Organización Internacional. El guion del panel de expertos se estructuró en tres bloques, centrados en preguntas relacionadas con los objetivos de la investigación. 
Tabla 1.

Preguntas del guion del panel de expertos

¿Qué es o en qué consiste la integración TIC en las escuelas?

¿Están suficientemente integradas las TIC en las escuelas?

¿Qué beneficios aporta la capacitación en competencias digitales de los niños y jóvenes?

¿Supone algún peligro la integración de las TIC en las escuelas?, ¿cuáles?

¿Qué obstáculos detecta en el uso de las TIC en las aulas?

Fuente: elaboración propia

Uno de los actores principales a la hora de incorporar las TIC en los colegios son los directores o los equipos directivos de los centros escolares. Sobre ellos recae la responsabilidad de decidir, en gran medida, la dotación tecnológica de la que dispondrá el colegio, de qué tipo, en qué cantidad, así como la formación que recibirán los profesores, y por tanto los alumnos en relación con las TIC. Para ello, se planificaron entrevistas cualitativas (Oltmann, 2016) a miembros de los equipos directivos de centros escolares de Madrid con las que se pretendía conocer sus opiniones sobre la importancia que les conceden a las TIC, el lugar que ocupan en su proyecto educativo, sus apuestas por la formación del claustro de profesores y sus actitudes hacia las TIC puesto que resultan de vital importancia para conocer realmente si se están integrando en la práctica docente.

Para seleccionar los centros se realizó un muestreo polietápico estratificado por conglomerados por niveles de enseñanza (infantil/primaria/ESO), tipología de centro educativo (pública o privada/concertada) y nivel socioeconómico del distrito en el que se encuentra el colegio, adoptando como marco de la encuesta el listado de centros que recoge la página web de la Consejería de Educación de la Comunidad de Madrid. Se realizaron las entrevistas al equipo directivo de los siguientes centros: colegio concertado Patrocinio de María, colegio concertado Tajamar, colegio concertado Pureza de María, Centro público Juan de la Cierva, Instituto de Enseñanza Secundaria Santamarca y colegio privado Santa Helena. Se plantearon las siguientes cuestiones en el guion de las entrevistas:

\section{Tabla 2.}

Preguntas del guion de las entrevistas a los equipos directivos

¿Cuál es su percepción de las TIC como directora del colegio?, ¿apuesta por ellas?

¿Cuándo empezó el colegio a implantar políticas TIC en sus prácticas docentes? pasos y elementos: número de ordenadores, conexión wifi, costes...

¿Disponen de tablets u ordenadores para todos los alumnos o de un aula de ordenadores/ informática donde acuden los estudiantes? Si tiene aula, ¿cada cuánto la utilizan?, ¿cuántos puestos tiene?

¿En qué cursos utilizan las nuevas tecnologías? 
En estos cursos, ¿las usan en todas las asignaturas o solo en algunas?, ¿en cuáles?

¿Piensan ampliar su uso a todos los cursos y a todas las clases?

¿Qué beneficios encuentran en usar las TIC en las clases?

¿Se encontró con problemas cuando empezó a implantar su uso en el colegio?, ¿cuáles? logísticas, queja de los profesores...

¿Los alumnos hacen los deberes en casa a través de alguna plataforma o aplicación que luego envían a los profesores? o ¿los hacen en papel y se los corrigen los profesores en clase?

¿Sus profesores tienen blog en el colegio?, ¿De qué asignaturas?, ¿cada cuánto los actualizan?

¿Desde la dirección se les incentiva a abrirlo y mantenerlo activo?

¿Los alumnos fuera del colegio se comunican con sus profesores vía mail o a través del blog de los profesores?

¿Considera que las TIC están ayudando a mejorar las prácticas docentes (las clases) de sus profesores? ¿En qué aspectos? Tres o cuatro

¿Permiten sus profesores que los alumnos tengan encendidos los teléfonos móviles en clase?

¿En las clases los estudiantes trabajan con sus móviles, con ordenadores o tablets que pone el colegio a disposición de los alumnos?

¿Nota a sus alumnos más motivados?, ¿ha mejorado su participación activa en clase?, ¿hacen más los deberes?

\section{RESULTADOS}

\subsection{Panel de expertos, autonomía y empoderamiento del profesorado mediante la formación}

Todos los participantes del panel de expertos se muestran críticos con la realidad de la implantación de las TIC en los centros escolares. En primer lugar, se abordó con ellos cuál es su percepción sobre el éxito de dicha implantación en las escuelas y todos señalaron que la integración de las TIC en el sistema escolar se está produciendo de forma más lenta de lo esperado. No obstante, reconocen que desde las Administraciones Públicas se están realizando esfuerzos e importantes inversiones para adquirir recursos tecnológicos para ponerlos a disposición del profesorado, pero, de nuevo, todos ellos señalan que estas iniciativas no han venido de la mano de inversiones en pedagogía, por lo que no están resultado muy efectivas:

"Está superbién la tecnología, pero antes de eso hay que pensar una serie de competencias, una serie de metodologías, una pedagogía nueva".

"Yo creo que la base de todo es la pedagogía y la metodología, y da igual si estamos enseñando competencia digital, competencia lingüística, CLIL o lo que sea". 
Afirman que el sistema educativo no es que necesite un cambio "necesita una revolución" para poner el poder en las manos de los equipos directivos y de los profesores. Indican que deberían ser estos agentes sociales los que lideren esta transformación, porque en la toma decisiones participa:

"Gente que no tienen la menor idea de un aula, que nunca ha estado en ningún aula, que no sabe nada sobre la pedagogía, sobre la psicología de los niños o los estudiantes".

Señalan que los estamentos gubernamentales están legislando y participando en el diseño de los proyectos de implantación de las TIC, pero que no están presentes en el día a día de las escuelas, por lo que no conocen la problemática real de los centros escolares. Uno de ellos afirma que lo que se está haciendo es "poner parches" tanto en la escuela pública como en la concertada y todos coinciden en que, aunque existen colegios que están poniendo en marcha proyectos para integrar las TIC, desde los organismos públicos falta una proyección más didáctica, que debe ir más allá de la simple equipación tecnológica de las aulas.

Los expertos coinciden en señalar que las editoriales son corresponsables de esta situación e indican que las editoriales en general, salvo algunas excepciones, simplemente están pasando el libro de papel a PDF o a otro formato electrónico sin modificar los contenidos, la manera de presentarlos o la forma de enseñarles esos contenidos a los alumnos. Manifiestan que el sistema educativo ha creado un sistema industrial de enseñanza en el que los contenidos provienen de las Administraciones Públicas para llegar a los colegios, cuando debería resolverse al revés. Afirman que los profesores y los directores de los centros escolares son los que deberían trabajar en los contenidos curriculares y:

"Hasta que no se cambie eso, creo que vamos a seguir yendo a remolque e intentando poner nuevas leyes".

Indican que estos cambios no se están produciendo porque supondría introducir nuevos modelos educativos que amenazan a las figuras constituidas desde el poder:

"Es decir, estamos hablando, yo diría, de introducirnos en el epicentro de los cambios radicales o son cambios que habrá que empezar a confrontar, estructurales".

Los expertos plantean la búsqueda de soluciones para que la implantación de las TIC, desde el punto de vista didáctico, sea realmente un éxito y apuestan por aconsejar que en los colegios se deberían enseñar competencias digitales como materia transversal en todas las asignaturas. Para ello apuestan por empoderar al profesor: 
"La competencia digital en la educación son habilidades del profesor y luego son habilidades transversales que mejoran la pedagogía, la competencia lingüística, la competencia matemática, la competencia social. Es decir, la competencia digital son habilidades transversales. Y la competencia digital no es saber lo que es el ciberbullying, es saber dar respuesta a las situaciones que se te van a ir dando en el colegio. ¿Y quién lo tiene que enseñar? El profesor."

Para que los profesores puedan enseñar y puedan formar a los alumnos en esta competencia, vuelven de nuevo sus ojos hacia las Administraciones Públicas que son las responsables de incluir estas enseñanzas en el currículo y uno de ellos, profesor con experiencia desde hace más de 25 años, indica que:

"Si tengo que meterlo curricularmente necesito un diseño curricular, y si yo necesito un diseño curricular, necesito formar a los profesores para que puedan impartir ese currículum, escuelas universitarias, facultades de Educación, que existe este currículum, que tienen que ustedes desarrollar las escuelas, perfectamente estratificado desde Infantil hasta Bachillerato, con unos contenidos en el cual cuando el niño esté en $3^{\circ}$ de Primaria va a ver unas cosas, que en $4^{\circ}$ va a ver otras y que desde el principio hasta el final, o sea, esos 15 años de formación básica el niño salga a la sociedad con una formación digital".

La escasa formación TIC del profesorado se revela como uno de los hándicaps más relevantes para que las competencias digitales se integren en el currículo de los estudiantes y que desde las Administraciones Públicas no se le está otorgando la suficiente importancia. Los expertos inciden en numerosas ocasiones a lo largo del panel en la necesidad de formar correctamente en competencias digitales al claustro docente:

"No creo que estemos dando a los profesores suficiente formación. Creo que muchas instituciones en lugar de ver la formación de los profesores como una inversión, lo ven como un gasto y es para cumplir el expediente, o sea, ya hemos hecho una jornada de formación, hasta el año que viene y no hay continuidad. Entonces, sale el profesor por la puerta con cinco actividades o diez y, después de dos semanas, ya ha acabado. Para mí una de las cosas más importantes en esto es el tema pedagógico, conocer cómo las utilizamos".

Si los directores y profesores adquiriesen, con más formación, nuevas competencias digitales serían ellos los que podrían generar sus propios contenidos sin tener que depender de los que les ofrecen las editoriales. Tanto es así, que uno de los expertos afirma que la competencia digital docente puede hacer desaparecer a las editoriales:

"Un profesor que tenga la suficiente capacitación, mediante un blog o mediante una herramienta, un LMS que le preste su colegio, puede tener su propio libro con contenido curricular". 
Pero para poder crear contenidos, vuelven a priorizar la necesidad de la formación docente:

“¿Quién enseña al profesor lo que puede y lo que no se puede integrar?, ¿cómo sabe un profesor lo que puede subir, lo que puede compartir, con qué puede colaborar? No se lo enseña nadie. Por lo que la formación competencial de los docentes continúa resultando insuficiente para poder dar un paso más en la integración de las TIC en los modelos didácticos de aprendizaje”.

Los expertos añaden que también existen deficiencias con la equipación tecnológica. El proyecto de conectividad es un proyecto de la Agenda Digital Europea pero en España está todavía en fase inicial. Ponen de manifiesto que aunque se han realizado avances en equipación tecnológica, persisten problemas técnicos que aún no se han resuelto. Los colegios no están adaptados a las necesidades para que las TIC puedan usarse en las aulas y ante el intento de implantación de un proyecto de mochila digital se evidenció que:

"La conectividad de calidad en España es una utopía, salvo en algunos colegios de grandes ciudades (...) para que un colegio tenga mochila digital tiene que tener una infraestructura (...) tiene que tener unos sistemas de wifi muy potentes, tiene que tener unos sistemas de continuidad preparados, tiene que cambiar toda la instalación eléctrica, son tantas las cosas que tiene que tener".

Entre los motivos que alegan se centran en señalar las diferencias existentes entre comunidades autónomas. De hecho, uno se pregunta:

“¿Por qué tenemos que tener un sistema educativo a nivel de infraestructura tecnológica tercermundista y una infraestructura de formación que es muy dispar, dependiendo de dónde estés como profesor, en qué grupo de colegios o en qué comunidad estés?".

Para que la equipación tecnológica de los centros escolares mejore y que la formación en competencias TIC logre incrementarse, los expertos del panel señalan a la dirección de los centros educativos como un pilar fundamental y garante sobre el que pivotan las decisiones relativas al fomento, visibilidad y mayor cobertura del uso de las TIC en las aulas. Los expertos afirman que por mucha alfabetización digital que se le dé a un profesor o por muchos ordenadores o tablets que se compren, si el equipo directivo no apuesta por poner en marcha programas centrados en las TIC no podrán dar el siguiente paso para convertirse en un colegio basado en el desarrollo de competencias digitales para el profesorado y los alumnos. 


\subsection{Entrevistas a equipos directivos de centros escolares, escasez de medios y falta de metodología didáctica}

En la segunda técnica de investigación se entrevistó a directores y responsables TIC de colegios de Madrid para comprobar si la implantación de las TIC se está produciendo gracias al impulso de las Administraciones Públicas y cómo se están llevando a cabo. El punto de partida para la implantación de la TIC en los colegios que participaron en el estudio coincide con la puesta en marcha por parte de las Administraciones Púbicas del Programa Escuela 2.0 cuya premisa era "un ordenador por estudiante". Así lo confirma uno de los entrevistados que, preguntado por la fecha de llegada de los ordenadores en el centro afirma que:

"Las TIC se comenzaron a usar en el centro hace unos quince años. $Y$ en la actualidad, hay tres salas de ordenadores con unos 18 ordenadores cada una de ellas".

Otro centro señala que:

"La Comunidad de Madrid nos dio hace por lo menos diez años un aula de informática y entonces empezamos con la informática como asignatura en $4^{\circ}$ de la ESO y a partir de ahí comenzamos con los ordenadores en las clases".

Y desde entonces, como declara el mismo centro, han ido adquiriendo dispositivos electrónicos hasta que en la actualidad disponen de:

"31 ordenadores y tres en infantil, o lo que en total serían 34, más otro que tienen los profesores lo que supone un total de 35 ordenadores, unos son portátiles y otros de mesa".

Lo que según el entrevistado resulta insuficiente puesto que solo existe un ordenador para todos los profesores del centro lo que hace muy complicado, si no imposible, que el profesorado puede hacer uso de las TIC para preparar sus clases en el colegio.

Todos los colegios, menos uno, reconocen que no disponen de ordenadores en las clases, sino que tienen la tradicional clase de informática a la que llevan a los alumnos. Este colegio que señalamos, el único que ha dado el salto tecnológico comenta que cuando comenzaron a informatizar su centro primero instalaron ordenadores y pizarras electrónicas y rápidamente se decantaron por el uso diario de las tablets como herramienta sobre la que se soportan todos los contenidos didácticos:

"Hace cinco o seis años empezamos poniendo pizarras digitales en el aula, porque había que cambiar un poco el modelo educativo, adaptado a las nuevas tecnologías. Pusimos pizarras, pusimos tres clases con pizarras, la experiencia fue buena. Al año siguiente se pusieron pizarras en otras tantas clases, pero 
después ya vino rápidamente el asunto de las tabletas, porque el propio proceso nos hizo decidirnos, y las editoriales también".

Para el resto, el proceso está siendo más arduo. Dos centros públicos reconocen que no es hasta hace unos cinco o seis años cuando empezaron a instaurar el uso de las TIC para su práctica docente diaria. El salto de los ordenadores al uso de las tablets lo han dado de forma dispar dependiendo de su poder adquisitivo y de la iniciativa del colegio. Uno de los centros resalta que:

"En el año 2011/2012 pusimos en marcha del Proyecto Tablet con el fin de acercar las nuevas tecnologías a las aulas. Este proyecto comenzó en Secundaria, en concreto en $1^{\circ}$ ESO y actualmente está funcionando en toda la etapa de Secundaria. Al finalizar el año académico el equipo directivo, junto con los coordinadores de los distintos departamentos, se reúnen y analizan si el proyecto debe continuar o no y ven los aspectos a mejorar".

Afirma que no están seguros si el Proyecto Tablet seguirá extendiéndose a la etapa de bachilleratos, porque todavía no han encontrado el equilibrio entre facilidades versus problemas que conllevan el uso de las TIC en sus aulas.

Otro de los centros cuenta que consiguieron dar el paso al uso de las tabletas en sus clases gracias a que ganaron un proyecto digital de la Comunidad de Madrid:
"Salió un proyecto de la Comunidad de Madrid que durante un plazo podías presentar un proyecto para tener nuevas tecnologías en el cole, y entonces el equipo directivo nos propuso a algún grupo de profesores que si queríamos presentar el proyecto, lo titulamos de las TIC a las TAC y cuando volvimos en septiembre la directora una día nos dijo que se nos había ido un poco de las manos porque habíamos sido elegidos dentro de ciento y pico colegios. Habíamos ganado 90.000 euros en una dotación digital. Gracias a eso pusimos en el cole puntos WIFI. Entonces, nos mandaron 160 tabletas divididas en cuatro carros con 40 tabletas".

La falta de medios es una constante en todos los colegios, excepto en el que dispone de unas infraestructuras TIC muy desarrolladas que pueden soportar la conexión de sus alumnos a través de Internet. El resto, para poder desarrollar sus políticas TIC, demandan a los organismos oficiales más medios para disponer de mayor dotación tecnológica y actualizar la que poseen.

Este colegio concertado, más desarrollado desde el punto de vista TIC, usa las tablets para todo su contenido curricular. Señala que el proceso para instalar las tabletas en todos los cursos no fue tan fácil ya que implicó un cambio de mentalidad en los profesores y en los padres. Para los niños el proceso de adaptación fue rápido: 
"Se fue convenciendo a los padres porque progresivamente se fue incorporando. Se hicieron reuniones para informar a los padres. Se contó con el apoyo de la mayoría, anecdóticamente te voy a decir, el primer año se dejó tanta libertad a las familias para elegirlo que de las cuatro clases que había en ese primer curso una era sin iPad, o sea, de las cuatro, tres tenían iPad y una no. Los que no tenían iPad van a esas clases".

El mismo centro afirma que lo que más costó fue adaptar la metodología didáctica a estos nuevos recursos. Sin embargo, su implantación ha sido tan efectiva que los alumnos no estudian ni trabajan con libros en papel, aunque sí utilizan cuadernos. En este caso, alumnos y profesores trabajan con las tablets y su aula de ordenadores no se utiliza para uso curriculares, como ocurre en el resto de los colegios:

"En el colegio hay algunas aulas de ordenadores que utilizan los alumnos en actividades extraescolares, por ejemplo, robótica pues utilizan ordenadores porque tienen que utilizar programas de diseño, de creación de elementos, pero es para lo único que utilizan".

Los demás centros escolares también disponen de ordenadores y de aulas de informática, pero su uso es curricular y varía dependiendo de la madurez tecnológica del centro. Es decir, unos centros utilizan las aulas de informática o tecnología como apoyo a la educación tradicional basada en los libros en papel, disponen en sus horarios de la asignatura de informática; otros usan las tablet que pone a disposición el centro escolar, pero sufragadas mediante un canon por los progenitores; otro les deja a los alumnos las tablets financiadas por la Comunidad de Madrid y otros, son los padres de los menores los que las compran y los niños las llevan a clase.

Uno de los aspectos más relevantes que señalan los directores y responsables TIC en el uso de las nuevas tecnologías es la capacitación de la que disponen los profesores. Todos los centros ofrecen cursos y seminarios para que el profesorado se forme ya sea costeado por la Comunidad de Madrid o por el colegio mismo. El responsable de un centro escolar afirma que dar el paso al uso de las TIC ha sido más complicado para su centro y han necesitado modificar la mentalidad del profesorado, más allá de los cursos y seminarios. Pone como ejemplo que cuando instalaron las pizarras digitales todavía no habían entendido bien su uso y sus ventajas:

"La primera fase de las pizarras digitales en el cole no cogimos bien el concepto de lo que era una pizarra digital, entendíamos que era una pizarra de verde que era blanca, y entonces lo usábamos solo y exclusivamente para escribir en su gran mayoría".

El mismo responsable señala que la formación del profesorado ha tenido que ser gradual e incluso específica para cada necesidad concreta de cada aula. Al principio, impartieron una formación global para todo el profesorado con charlas a las que 
invitaban a ponentes sobre cómo hacer presentaciones, cursos de pizarra digital, cómo manejar Internet, pero el nivel de sus profesores era dispar, lo que ocasionó problemas respecto la información que debían aportar estos seminarios. Unos profesores ponían mucho interés en mejorar, otros necesitaban una formación más básica porque no entendían lo que se les estaba enseñando, lo cual bajaba el nivel de los cursos y otros simplemente no querían aprender. Al final, el colegio creó:

"Un seminario que hemos hecho dentro del centro, sin que venga nadie de fuera, y lo que estamos haciendo es trabajar un poco entorno a las necesidades de cada grupo según su nivel".

Tal y como declara uno de los responsables TIC, algunos profesores no se muestran predispuestos y proclives a la continua actualización que exige el uso de las TIC y señala que, aunque anualmente les ofrecen cursos:

"Supone un esfuerzo para los docentes ya que se trata de actualizar constantemente contenidos".

Esta escasa predisposición incrementa la brecha digital entre profesores y alumnos, y entre profesores que desean formarse en TIC y aquellos que, por diversos motivos, han desistido de hacerlo. Solo uno de los responsables entrevistados afirma que no depende tanto de la edad del docente como de su interés por renovarse:

"Ha habido una época en que sí había tres o cuatro profesores mayores que estaban a punto de jubilarse y algunos de ellos no lo han intentado siquiera".

En el resto de los centros se detectan que los alumnos y los profesores más jóvenes presentan una actitud más activa hacia el uso de las TIC.

A los niños les gustan mucho las TIC, les entran por los ojos. Además como ellos son de la era de las nuevas tecnologías las resulta más fácil y además no solo para los alumnos también para los profesores son importantes.

En esta línea, otro afirma que los alumnos las dominan mejor y que, sin duda, las tienen más integradas en su vida diaria. Otro responsable afirma que ellos no notan excesivamente la brecha digital por la edad, subraya que depende más de la actitud y de la predisposición:

"En un rango de 30 a 40 años que se cierra porque no está y no le apetece, porque ellos dicen que no están capacitados y luego tenemos gente la cual se va a jubilar. Y luego tenemos gente pues eso de 50 y pico años que incluso está yendo a cursos de fuera del cole". 


\section{DISCUSIÓN Y CONCLUSIONES}

Ciuffolini y Hernández Bertone (2017) proponen analizar las políticas públicas desde su configuración, gestión y regulación puesto que afectan al orden de las relaciones sociales. Esta implicación se pone de manifiesto en las dos dimensiones que adquiere la integración TIC en las escuelas. La primera es una dimensión tecnológica basada en la introducción de las TIC y la segunda de carácter pedagógico que no depende de los dispositivos electrónicos utilizados, sino de los modelos didácticos que se apliquen para mejorar la enseñanza (Tedesco, 2012). Estos modelos pedagógicos son los que marcan la diferencia en la incorporación de las TIC en la educación y deben ir acompañados del desarrollo del profesorado en la adquisición de competencias digitales y de contenidos relevantes de calidad (Toribio, Garbarini \& Fernández, 2016). Se confirma el objetivo de la investigación puesto que se recogen los proyectos impulsados por las Administraciones Públicas y sus resultados para democratizar las TIC en las aulas puesto que tal y como reconocen los expertos y directores de centros educativos y responsables TIC, la importancia de la responsabilidad de las Administraciones Públicas es clave para la implantación de las TIC en los centros escolares.

Los directores y expertos participantes en el panel afirman que la llegada de los ordenadores ha sido gracias a la financiación y apoyo de las Administraciones Públicas, no obstante declaran que resultan insuficientes desde el punto de vista de la equipación, innovación metodológica y formación digital de los docentes. Además, como afirman los expertos participantes en el panel, estas diferencias son más notables puesto que el despliegue tecnológico no se está desarrollando de manera homogénea debido a que las competencias educativas están transferidas a cada comunidad autónoma.

Los expertos señalan que se debe trabajar en la adquisición de competencias digitales de los padres y del profesorado con el objetivo de incrementar su capacidad de utilizar las TIC con fines educativos. Los equipos directivos y los expertos se muestran críticos ante formación competencial del profesorado que consideran insuficiente y acerca de la necesitad de equipar más a los colegios pero sobre todo de invertir en metodología y pedagogía ya que las TIC se están utilizando como apoyo al modelo tradicional de enseñanza pero no como un nuevo modo de entender la educación de los menores.

El último plan puesto en marcha a iniciativa del gobierno español fue el 'Programa Escuela 2.0.', que acercó los ordenadores a los alumnos y ofreció materiales virtuales a los docentes, pero no les proveyó de la formación metodológica necesaria para utilizarlos de manera eficaz. Este programa inaugurado en 2009 finalizó en 2011, hace 9 años. Desde entonces, España se ha guiado por la Agenda Digital Europea y ha adaptado sus requisitos a nuestro país en la Agenda Digital para España intentado popularizar el uso de las TIC en los sectores sociales más desfavorecidos y en la formación de nuevos profesionales TIC. 
Para mejorar el sistema educativo se revela necesario instaurar planes para fomentar la integración de las TIC, pero desde nuevas perspectivas teórico-prácticas. Se precisa que las Administraciones Públicas tomen medidas dirigidas a empoderar a la dirección y al profesorado. Para lograrlo es necesario incrementar la equipación TIC y mejorar la formación de los docentes en metodologías didácticas. De este modo, se logrará revertir la actual situación en la que las TIC no se han convertido en una herramienta renovadora de la didáctica en los centros educativos, ni los docentes disponen de una formación competencial acorde con la realidad que se vive fuera de las aulas.

\section{Referencias}

Albuquerque, C. P. (2017). Analítica da implementação de políticas públicas: aproximações construtivistas e hermenêuticas. Ámbitos. Revista Internacional de Comunicación, 39. doi:10.12795/Ambitos.2018.i39.07

Agius, S. J. (2013). Qualitative research: its value and applicability. The Psychiatrist, 37(6), 204206. doi:10.1192/pb.bp. 113.042770

Area, M., Alonso, C., Correa, J. M., Del Moral, M. E., De Pablos, J., Paredes, J., \& Valverde, J. (2014). Las políticas educativas TIC en España después del Programa Escuela 2.0: las tendencias que emergen. RELATEC. Revista Latinoamericana de Tecnología Educativa, 13 (2), 11-33. doi:10.17398/1695-288X.13.2.11

Bley, S. J., Hametner, M., Dimitrova, A., Ruech, R., De Rocchi, A., Gschwend, E., \& Umpfenbach, K. (2018). Smarter, greener, more inclusive. Indicators to support the Europe, 2020. Recuperado de https://bit.ly/38Mjj7l

Ciuffolini, M. A., \& Hernández Bertone, J. (2017). Una lectura posible de las políticas públicas. Decisiones teóricas, empíricas, metodológicas y epistemológicas para su análisis. Ámbitos. Revista Internacional de Comunicación, 39. doi:10.12795/Ambitos.2018.i39.10

Courts, B., \& Tucker, J. (2012). Using Technology to Create a Dynamic Classroom Experience. Journal of College Teaching \& Learning (TLC), 9(2), 121-128. doi:10.19030/tlc.v9i2.6907

Dror, Y. (2017). Public policy making reexamined. London: Routledge.

European Commission. (2010). Communication from the Commission to the European Parliament, the Council, the European Economic and Social Committee and the Committee of the Regions. A Digital Agenda for Europe. Brussels. https://bit.ly/2Wi1wTh

Fernández Fernández, I (2010). Las TIC en el ámbito educativo. Recuperado de https://bit.ly/1Tp3BST

Fundación Telefónica. (2012). Alfabetización digital y competencias informacionales (Vol. 20). Madrid. Fundación Telefónica. 
Gómez, L., \& Macedo, J. (2010). Importancia de las TIC en la educación básica regular. Investigación educativa, 14(25), 209-224.

García-Muñoz, S. C., Valiente, M. M., Barreiro, M. C., Díaz-Maroto, I. T., \& Rumayor, A. A. (2009). RedTIC-Red de Centros Educativos Avanzados en el uso de las TIC. Revista de Educación a Distancia. Recuperado de https://bit.ly/38TW5MJ

Hill, M., \& Hupe, P. (2014). Implementing public policy: An introduction to the study of operational governance. London: Sage.

Ivankovich-Guillén, C. \& Araya-Quesada, Y. (2011). Focus group: técnica de investigación cualitativa en investigación de mercados. Ciencias Económicas, 29(1), 545-554.

McKnight, K., O'Malley, K., Ruzic, R., Horsley, M. K., Franey, J. J., \& Bassett, K. (2016). Teaching in a digital age: How educators use technology to improve student learning. Journal of research on technology in education, 48(3), 194-211. doi:10.1080/15391523.2016.1175856

Kozma, R. B., \& Isaacs, S. (Eds.). (2011). Transforming education: The power of ICT policies. France: Unesco.

Krueger, R. A. (2014). Focus groups: A practical guide for applied research. London: Sage.

Knill, C., \& Tosun, J. (2012). Public policy: A new introduction. New York: Macmillan International Higher Education.

López, L. M., \& Martínez, J. P. (2017). Políticas públicas de fomento de la sociedad de la información en Europa y España (2000-2017). Panorama social, 35. https://bit.ly/2VBX4ul

Martín-Laborda, D. A, R. (2005). Las nuevas tecnologías en la educación. Madrid. Fundación AUNA. Recuperado de https://bit.ly/2wZOajK

Ministerio de Ciencia y Tecnología (2009). España.es Programa de Actuaciones para el Desarrollo de la Sociedad de la Información en España. https://bit.ly/2VGLZrZ

Ministerio de Industria, Comercio y Turismo (2015). Informe Anual de la Agenda Digital para España. https://bit.ly/2xOpt79

Moreira, M. A. (2002). La integración escolar de las nuevas tecnologías. Entre el deseo y la realidad. Revista del Fórum Europeo de Administradores de la Educación, 10(6), 14-18. https://bit.ly/2Yl8Mp7

Muñoz-Repiso, A. G. V., Gómez-Pablos, V. B., \& García, C. L. (2014). Las TIC en el aprendizaje colaborativo en el aula de Primaria y Secundaria. Comunicar: Revista científica iberoamericana de comunicación y educación, (42), 65-74. doi:10.3916/C42-2014-06

Murillo, F. (2010). Métodos de investigación en educación especial. España, Tercera Edición Educación Especial. Recuperado de https://bit.ly/2TWn4l6 
Oltmann, S. (2016). Qualitative interviews: A methodological discussion of the interviewer and respondent contexts. In Forum Qualitative Sozialforschung/Forum: Qualitative Social Research (Vol. 17, No. 2). doi:10.17169/fqs-17.2.2551

Organización de las Naciones Unidas para la Educación, la Ciencia y la Cultura. UNESCO (2019). Las TIC en la educación. Recuperado de https://bit.ly/2TMvDQD

Sangra, A., \& González-Sanmamed, M. (2011). The Role of Information and Communication Technologies in Improving Teaching and Learning Processes in Primary and Secondary Schools. Journal of Asynchronous Learning Networks, 15(4), 47-59. doi:10.14742/ajet.1020

Sandín Esteban, M. (2003). La enseñanza de la investigación cualitativa. Revista de Enseñanza Universitaria, 21, 37-52. Recuperado de https://bit.ly/2QkGiQB

Sanz, A. P. (2011). Escuela 2.0: Educación para el mundo digital. Revista de estudios de juventud, (92), 63-86.

Tedesco, J. C. (2012). Educación y justicia social en América Latina (No. 370 T256e). Buenos Aires, AR: Fondo de Cultura Económica, 2012.

Toribio, D., Garbarini, L., \& Fernández, A. (2016). El uso pedagógico de las TIC en Educación. https://bit.ly/2HFO2aG

Tur-Porcar, A. M. (2017). Crianza, competencia parental y su relación con el desarrollo de los hijos. Revista Mexicana de Investigación en Psicología, 6(2), 186-191. Recuperado de https://bit.ly/3hjgobW 